\title{
Specific Effects of an Amnesic Drug: Effect of Lorazepam on Study Time Allocation and on Judgment of Learning
}

\author{
Marie Izaute*,I and Elisabeth Bacon ${ }^{2}$ \\ 'Laboratoire de Psychologie Sociale de la Cognition (LAPSCO - UMR 6024 CNRS), Universite Blaise Pascal, Clermont-Ferrand Cedex, France; \\ 2INSERM U405, Clinique psychiatrique, Hopital civil, Strasbourg Cedex, France
}

\begin{abstract}
We investigated the effects of lorazepam, a benzodiazepine, on the allocation of study time, memory, and judgment of learning, in a cognitive task where the repetition of word presentation was manipulated. The aim was to assess whether lorazepam would affect the learning processes and/or whether the participants would be aware of the amnesic difficulty. A total of 30 healthy volunteers participated in the study, I 5 of whom received a capsule containing the lorazepam drug $(0.038 \mathrm{mg} / \mathrm{kg})$ and I 5 a placebo capsule. First, the accuracy of delayed judgments of learning $(\mathrm{JOL})$ was measured in both groups. For the $\mathrm{JOL}$ ratings, results showed that all the participants benefited from word repetition. Although the overall performance was lower in the lorazepam than in the placebo group, the accuracy of the JOL ratings was preserved by the drug. Second, all the participants benefited from the repetition of learning, although the performances of the lorazepam-treated subjects remained lower than those of the placebo participants. The repetition of learning had an effect on JOL in both groups. Finally, the time spent learning each (allocation study time) pair of words was measured. For the placebo group, results revealed that study time decreased significantly with the frequency of presentation. For the lorazepam group, no effect of presentation frequency was found. Overall, our findings suggest that the lorazepam drug has a differential effect on the monitoring and the control processes involved in a learning task. The implications of these findings are discussed within the theoretical framework of metacognition. Neuropsychopharmacology (2005) 30, 196-204, advance online publication, I 3 October 2004; doi: I 0. I038/sj.npp. I 300564
\end{abstract}

Keywords: benzodiazepines; lorazepam; study time; judgment of learning; metamemory

\section{INTRODUCTION}

Memory and metamemory deficits seem to be characteristic of benzodiazepine-treated participants. The cognitive effects of the benzodiazepine lorazepam have been widely investigated. Indeed, the fact that the lorazepam drug induces a transient amnesia following a single intake has been repeatedly shown (Allen et al, 1993; Bishop and Curran, 1998; Curran et al, 1993; Danion, 1994; Danion et al, 1989, 1992; File et al, 1992; Legrand et al, 1995; Pompéia et al, 1996; Vidailhet et al, 1996; Weingartner et al, 1993). During the amnesic episode, results have shown that episodic memory is specifically impaired (File et al, 1992; Fluck et al, 1998; Green et al, 1996; Duka et al, 1996; Curran 1991, 1999; Blin et al, 2001) and that it is independent of the benzodiazepine-induced sedative effects (Mintzer and

* Correspondence: Dr M Izaute, Laboratoire de Psychologie Sociale de la Cognition (LAPSCO - UMR 6024 CNRS), Universite Blaise Pascal, 34, Avenue Carnot 63037, Clermont-Ferrand Cedex, France,

Tel: + 3304734062 55, Fax: + 3304734064 82,

E-mail: Marie.lzaute@srvpsy.univ-bpclermont.fr

Received 19 December 2003; revised I July 2004; accepted I 3 July 2004

Online publication: 12 August 2004 at http://www.acnp.org/citations/ Npp08120403568/default.pdf
Griffiths, 2003a; Huron et al, 2002). Interestingly, participants do not seem to be aware of their memory deficits (Curran et al, 1987). In fact, participants treated with triazolam, another benzodiazepine, have been reported to be as certain as placebo participants of their capacity to recall words learned under the influence of the drug even if their recall performance is significantly impaired (Weingartner et al, 1993).

These previous studies all have in common the fact that they investigated global memory performances and not metamemory, that is, the participants' awareness of their own memory capacity and the control of the related cognitive behaviors (for a review, see for example, Nelson, 1996a). Metamemory can be thought of as a regulatory system by which to-be-learned items are mastered. Previous studies conducted in normal participants have confirmed that two important processes, monitoring and control, might influence memory performance (Nelson and Narens, 1990; Koriat and Goldsmith, 1996). Monitoring refers to the participant's subjective reports about his or her introspection, and is expressed as metamemory judgments such as judgment of learning (JOL) at the end of the acquisition session, and feeling of knowing (FOK) or confidence level (CL) at the time of retrieval. Control refers to anything that will modify the behavior of the participants, for example, 
allocate a given study time, produce an answer or abstain, continue an action or spend more time searching for a known information, etc.

In studies investigating metamemory, the experiments are typically based on the calculation of the correspondence between the accuracy of an answer and its metamemory rating. In the case of memory retrieval, Bacon et al (1998) were the first to investigate the effects of lorazepam on memory accuracy and metamemory rating. Their results indicated that the lorazepam drug impaired the metamemory processes, the semantic ones, and also the metamemory processes related to episodic memory. For the later, the items consisted of 50 short sentences devised so that the last word of each sentence was not predictable. Participants were asked to read each sentence aloud and following a 10min break, their task was to retrieve the last missing word of each presented sentence. If an answer was given, participants were then invited to express a CL on the accuracy of the answers provided. If no answer was given, a FOK prediction with regard to the future recognition of these nonrecalled items was recorded. Results revealed that lorazepam-treated participants exhibited both memory and metamemory impairments during the recall phase of the task. They provided significantly fewer answers and the accuracy of their confidence ratings was significantly lower than that observed in placebo participants. Furthermore, the predictive accuracy of their FOK ratings on a future recognition was at chance. The fact that the lorazepam drug impairs those metamemory processes that are activated to retrieve a word from episodic memory is consistent with reported clinical data. Mintzer and Griffiths (2003b) observed an impairment of metamemory ratings. However, to our knowledge, the effects of benzodiazepines on the metamemory processes involved both in the monitoring ability and the strategic allocation of the time of encoding have not yet been explored.

This article is specifically concerned with JOL ratings, that is, the explicit judgments given by the participants at the end of a learning procedure with regard to the likelihood of remembering recently acquired information on a subsequent memory test. Following a single learning trial, Mintzer and Griffiths (2003b) observed a preservation of JOL accuracy (as measured by relative accuracy measure) in conjunction with impaired memory, under the effects of lorazepam compared to placebo. Merritt et al (2003) observed that the mean JOL ratings were unaffected by the benzodiazepine midazolam following a single learning session, suggesting that participants had little insight into their memory deficits when asked to evaluate their future memory performance after encoding. Nelson et al (1998) found that acute alcohol intoxication impaired memory and participants were relatively unaware of this impairment during the study. As lorazepam and ethanol share common mechanisms of action through the GABA/benzodiazepine receptor (Ticku, 1983; Gonzales and Hoffman, 1991), one may expect that lorazepam could also induce some impairment in the monitoring ability at the time of encoding.

As the control of study time at encoding may be a critical feature of memory effectiveness, we also investigated how participants made use of an extrinsic cue, namely the repetition of learning trials, to adapt their learning strategies in terms of time allocated to the study (Koriat, 1997; Moulin et al, 2000b).

The focus of the present study was to explore the question of the respective implications of monitoring and control processes in the strategic regulation of memory accuracy in a learning task under the effects of lorazepam drug. First, two empirical measures that reflect metamemory functioning were compared: (1) delayed JOL ratings - an explicit measure of metamemory monitoring; and (2) allocation of study time - a measure of memory control (Nelson, 1993; Nelson et al, 1994). Second, we focused on one aspect of encoding behavior: the effect of repetition of stimuli. The present study concentrates in encoding factors in order to explore if lorazepam-treated participants benefited from repetition as much as controls (Moulin et al, 2000a,b). Following Koriat (1997), participants should be able to give an accurate judgement of their memory performance when the given cues are valid and, consequently, this would be an indication of their true memory performance (see also Mazzoni and Nelson, 1995). In fact, Koriat argues that participants predict their later memory performance from cues such as, for example, the ease with which an item is processed, the perceived difficulty of encoding that item or the frequency of presentation of that item. Consequently, we considered two possible repetition-based deficits that lorazepam-treated participants might show during encoding. First, they may be unaware of repetition and, hence, fail to act upon it. Second, they may be aware of repetition but fail to act appropriately. Benzodiazepines are usually considered to have no effect on short-term memory (for a review see Curran, 1999), and the effects of lorazepam itself seem to be somewhat mixed (Vermeeren et al, 1995; Patat et al, 1995; Mintzer and Griffith, 2003a, b). Consequently, we registered delayed JOLs, that is, JOLs expressed after a delay, in order to have the two measures, memory and metamemory, both made within long-term memory, a state in which lorazepam exerts its anterograde amnesic effect (Dunlosky and Nelson, 1994). This gave us the means to assess the effects of repetition of learning on memory, monitoring, as well as on monitoring effectiveness. Indeed, JOLs made after a delay following initial study are usually highly correlated with memory performance and more likely to be based on mnemonic factors (Nelson and Dunlosky, 1991; Kimball and Metcalfe, 2003).

\section{METHOD}

\section{Participants}

In all, 30 healthy volunteers (17 women and 13 men) were recruited from the University of Strasbourg (Faculty of Medicine, Pharmacy and Dentistry). All were French native speakers and were paid for their participation. Participants ranged in age from 20 to 27 years (mean: 22.8 years) and in weight from 51.6 to $79 \mathrm{~kg}$ (mean: $64.2 \mathrm{~kg}$ ). They were healthy and had no history of illness, alcoholism, drug abuse, or tobacco consumption of more than 10 cigarettes per day. There were no chronic benzodiazepine users and none of them had taken concomitant medication for at least 15 days prior to their participation. Participants with a high consumption of coffee, tea (more than three cups a day), or coca-cola were not included in the study. 
Participants were pseudorandomly assigned to one of two parallel groups: a placebo group $(n=15)$ and a lorazepam $0.038 \mathrm{mg} / \mathrm{kg}$ group $(n=15)$. The two groups did not significantly differ in age, $\mathrm{t}(28)=0.38, p>0.05$ or in weight, $\mathrm{t}(28)=0.71, p>0.05$. They did not differ either in their predrug general knowledge level as evaluated by the Information subtests (12.7 for the placebo group and 12.8 for the lorazepam group), $\mathrm{t}(28)=0.29, p=0.82$, and the Vocabulary subtests (11.27 and 11.3, respectively), $\mathrm{t}(28)=0.24, p=0.81$, of the Wechsler Adult Intelligence Scale revised (WAIS-R, Wechsler, 1987). Informed written consent was obtained from all the volunteers before they enrolled in the study, which was approved by the Faculty Ethics Committee. The participants were instructed to abstain from beverages containing caffeine and alcohol for a $24-\mathrm{h}$ period preceding the study.

\section{Stimuli}

The second author selected 60 pairs of words nonassociated (eg accordion-body/boar-telephone).

\section{Procedure}

The capsule was given orally using a double-blind procedure. The experimental session started $2 \mathrm{~h}$ after the intake of the drug (0730). Computerized versions of the tasks were used. The participants were tested individually in the presence of the experimenter. They sat in front of a computer screen and used the mouse key. They were instructed that pairs of words (item: cue/target) would be presented simultaneously and visually on a computer screen. The task was to recall as many target words as possible when presented with a cue. The pairs appeared one by one on the screen and the participants were required to read them aloud. They could study each pair for as long as they liked in order to maximize their chances of remembering the target later. Indeed, the items remained on the screen until the participant pressed the space bar to view the next pair of words. The study time was fixed to last between 2 and $8 \mathrm{~s}$. Participants were warned that some items would be presented two or three times. They were encouraged to treat these repetitions as an opportunity to better memorize the items. The computer measured the study time allocated to each item.

During the encoding phase of the task, the computer measured the study time allocated to each pair of words. With three levels of repetition for each item, participants were presented a total number of 120 pairs $(20 \times 1+20 \times 2$ $+20 \times 3)$. These pairs were presented in an identical fixed order for all participants. The repetition of one item was separated by a minimum of five other items.

After the encoding phase, a 4-min retention phase was occupied with a nonverbal distractor task (Kelemen and Weawer, 1997). This time interval was also used to provide detailed instructions on the delayed JOL ratings. After a detailed description of the task requirements, a concrete example was provided on the computer screen.

After the retention phase, cues for each item were presented without the target word. Participants were required to give their JOL ratings using a $100-\mathrm{mm}$ visual analogue scale that appeared on the screen. For example, if the item 'desk-ship' had been presented during the study, the JOL prompt was 'desk-' followed by the query, 'How confident are you that in a few minutes from now you will be able to recall the second word of the pair when prompted with the first?'. JOL ratings were defined starting at $' 0 \%=$ definitely won't recall' - on the left and ending off with ' $100 \%=$ definitely will recall' - on the right. The participants reported their estimate by clicking with the mouse on the visual analogue bar. Immediately after this phase with JOL ratings, there was a free recall test. During this test phase, the 60 pairs were presented on the screen and the participants had to either provide the target word or say, 'I don't know'. The experimenter entered the answer in the computer. Finally, participants were asked to rate their CL that the answer provided was correct. This was carried out once more using a $100-\mathrm{mm}$ visual analogue scale.

After completion of the entire procedure, the experimenter led an informal discussion during which the participants were asked to report their comments about the experiment, to describe the learning strategies that they may have used as well as to give a description of their overall memory performance in daily activities.

\section{Analog Self-Ratings of Sedation}

Halfway through the study, that is, $2.5 \mathrm{~h}$ after the intake of the capsule, participants used a set of 16 visual analog scales to assess their subjective state (Norris, 1971). Each scale consisted of a 100-mm nongraduated horizontal line, anchored by descriptions of contrasting states of mind. Participants were asked to rate their feelings at the time by placing a vertical mark across each horizontal line. After the end of the session, the scales were scored by measuring the length (in $\mathrm{mm}$ ) between the participants' mark and the positive end of each horizontal line. The mean score of nine of these scales (alert/drowsy; strong/feeble; muzzy/clearheaded; well coordinated/clumsy; lethargic/energetic; mentally slow/quick witted; attentive/dreamy; incompetent/ proficient; interested/bored) was taken as a measure of sedation (Bond and Lader, 1974). Sedation scores were significantly higher for the lorazepam group $(S=44.1$; $\mathrm{SD}=13.9)$ than for the placebo group $(S=22.0 ; \mathrm{SD}=18.4)$, $\mathrm{t}(26)=3, p<0.005$. A Pearson correlation analysis was conducted and revealed no significant correlation between the self-ratings of sedation, on the one hand, and the criterion for recall performance and JOL ratings, on the other. Results were similar for both the lorazepam and the placebo group.

\section{RESULTS}

In the following, we present the results for the effects of lorazepam on: (1) memory accuracy; (2) allocation of study time; (3) JOL ratings; and finally, (4) subjective evaluation and adopted strategy. Two word pairs among the material presented once at the learning session were removed from the calculations as they shared a common word.

\section{Memory Accuracy}

Recall performance. For each participant, the mean percentage of correct recall performance was calculated on 
the placebo and the lorazepam group, for each level of item repetition (1st, 2nd, 3rd presentation). Memory performance was analyzed by a $2 \times 3$ (group $\times$ repetition) repeated measures ANOVA. The statistical analysis revealed a main effect of group, $\mathrm{F}(1,28)=30.01, p<0.001$ : the percentage of correct recall was lower for the lorazepam group $(37.6 \%, \mathrm{SD}=31.9)$ than for the placebo group $(77.0 \%, \mathrm{SD}=22.2)$. There was a main effect of item repetition, $\mathrm{F}(2,56)=86.12, p<0.0001$, indicating that the percentage of correct recall was higher for words that had been presented two or three times than for those that had been presented only once (see Table 1). The interaction was not significant.

Proportion of commission errors. For each level of item repetition, the mean percentage of commission errors was calculated out of the total number of answers provided. There was a main effect of group, $\mathrm{F}(1,28)=16.81, p<0.001$, the percentage of commission errors was higher for the lorazepam group $(30.3 \%, \mathrm{SD}=27.9)$ than for the placebo group $(4.6 \%, \mathrm{SD}=8.9)$. There was also a main effect of repetition, $\mathrm{F}(2,56)=20.31, p<0.001$, which indicated that the percentage of commission errors was lower for those words that had been presented more frequently. Finally, the interaction was significant, $\mathrm{F}(2,56)=6.72, p<0.01$ : the lorazepam group produced significantly more commission errors for pairs of words that were presented once compared to those presented two or three times, $\mathrm{F}(1,56)=33.74, \quad p<0.001$; in the placebo group, the frequency of presentation had no reliable effect on the percentage of commissions errors (see Table 1).

\section{Allocation of Study Time}

There are two ways in which the study time can be analyzed. One can examine the means for each level of item repetition for all items (ie the 1st presentation of each word $v s$ the second presentation $v s$ the third presentation). This analysis was conducted first. This analysis confounded, however, pairs of words and presentation frequency. Indeed, we had 58 pairs in the group of pairs presented at least once, 38 pairs in the group of pairs shown at least twice, and 18 pairs were shown three times. Consequently, a second analysis was conducted in order to examine the effects of presentation frequency only for those items that had been presented three times (ie 18 pairs of words). For all dependent variables, results were similar for both analyses. Hence, we report only the statistical results for the latter analysis, which was conducted on 18 pairs of words.

Table 2 shows the time allocated to the study of each pair of words before recall, taking into account the effect of repetition. The main effect of Group did not reach significance. The main effect of Repetition did, $\mathrm{F}(1,28)=41.48, p<0.001$. Finally, the interaction was significant, $F(2,56)=18.20, p<0.001$. Although there was a main effect of Repetition, this effect was carried by the placebo group. Indeed, the placebo group spent more time exploring pairs of words on their first repetition than on their second repetition, $\mathrm{F}(1,56)=60.74 ; p<0.001$ and more time on their second repetition than on their third repetition $(\mathrm{F}(1,56)=6.03 ; p<0.02)$. The lorazepam group did not make this distinction and allocated the same amount of time whatever the presentation frequency (no difference between the first and the second repetition, $\mathrm{F}(1,56)=1.90 ; p=0.17$, and between the second and the third repetition, $\mathrm{F}(1,56)=0.63 ; p=0.43$, respectively). The two groups did not provide different allocated time for the first presentation, $\mathrm{F}(1,56)=0.004 ; p=0.95$. However, placebo participants produced a lower allocated time than lorazepam participants for the second and the third repetition $(\mathrm{F}(1,56)=41.96 ; p<0.001$ and $\mathrm{F}(1,56)=66.28$; $p<0.001$, respectively).

Table I Proportion of Correct Recall (CA) and Commission Errors (C) for Pairs of Words Presented One, Two, or Third Times

Drug group

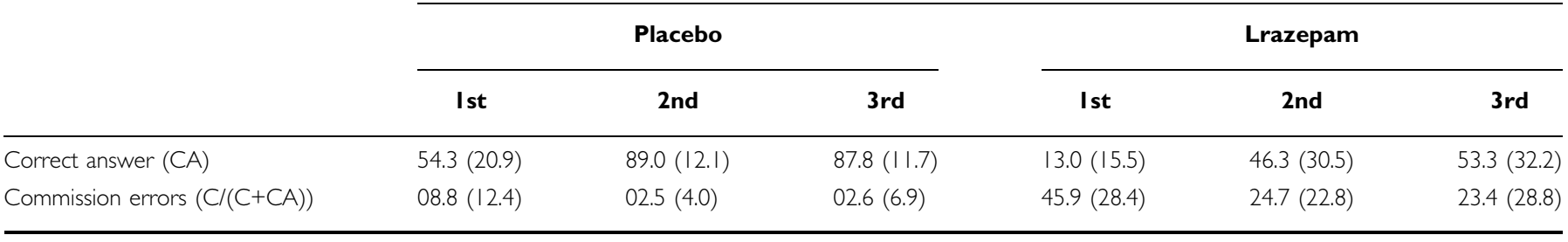

Table 2 Mean Time Allocated to the Study of Pairs of Words on Their Ist, 2nd, and 3rd Repetition

Drug group

\begin{tabular}{|c|c|c|c|c|c|c|}
\hline & \multicolumn{3}{|c|}{ Placebo } & \multicolumn{3}{|c|}{ Lorazepam } \\
\hline & I st & 2nd & $3 r d$ & Ist & 2nd & 3rd \\
\hline
\end{tabular}




\section{JOL Ratings}

Magnitude of the JOLs. The mean item-by-item judgments was calculated separately for each individual for delayed JOLs. Table 3 reports the mean JOL (and standard deviation) for items presented once, twice and three times.

The main effect of Group was significant, $F(1,28)=16.22$, $p<0.001$, which indicated that the lorazepam group was less confident in their future performance level $(53.5 \%$, $\mathrm{SEM}=2.93)$ than the placebo group $(75.6 \%, \mathrm{SEM}=3.70)$. There was a main effect of Repetition, $F(2,56)=104.20$, $p<0.0001$. All participants rated as easier the fact of recalling items that were presented more frequently. The interaction was not significant.

Accuracy of the JOL ratings (relative resolution). For each participant, a gamma coefficient was computed (GoodmanKruskal gamma correlation, Nelson, 1984, 1987) in order to gain a better description of the relative accuracy of the JOL ratings for each individual participant (Nelson, 1996b). The values of the gamma coefficient can range from 1.0 (in the case of a full agreement between JOL rating and the given answer, that is, a high JOL rating for a correct answer and a low JOL rating for a wrong answer) to -1.0 (in the case of a full disagreement between JOL rating and the given answer). Gamma correlations were computed for each participant, and the means of those correlations were analyzed as a function of drug group. Results revealed no reliable differences between the lorazepam group $(G=0.91$, $\mathrm{SD}=0.10)$ and the placebo group $(G=0.93, \mathrm{SD}=0.07)$. Neither the main effect of repetition nor the interaction reached significance.

Calibration (absolute resolution). Following the procedure commonly used in calibration studies (see Lichenstein et al, 1982), Figure 1 plots the percentage of correct recall against mean JOL ratings, with the probability judgments grouped into 10 preset intervals $(0-10$, 11-20...91-100; see Koriat, 1997). Perfect calibration is indicated by the diagonal line (Lichenstein et al, 1982). Results show that participants in the placebo group are relatively well calibrated. On the other hand, participants in the lorazepam group are characterized by a certain level of over confidence. This observation is coherent with the results presented so far, which indicate that participants in the lorazepam group give fewer correct answers, even if the accuracy of the JOL resolution is similar across groups.

\section{Subjective Evaluation and Adopted Strategy}

At the end of the experimental session, an informal discussion was conducted with the participant during which they were asked to report their comments about the study and to describe the learning strategies that they may have used. The two groups differed with some respect when considering their global insight about performance level. The placebo participants were more numerous to consider the task as easy: eight rated it as easy and five as difficult, whereas the inverse pattern was observed among the lorazepam group with only three participants having viewed it as easy and nine as difficult (the other evaluations were 'surprising', 'amusing', 'relative'). The experimental groups also differed in the global estimation of their performance: in the placebo group, six participants considered their performance as good, six as average, and none as bad. The lorazepam participants were more pessimistic with two individuals having considered their performance as good, five as average, and eight as bad or even terrible. When considering the strategies adopted, 13 placebo participants described using mental imagery, visualization of the set-up,

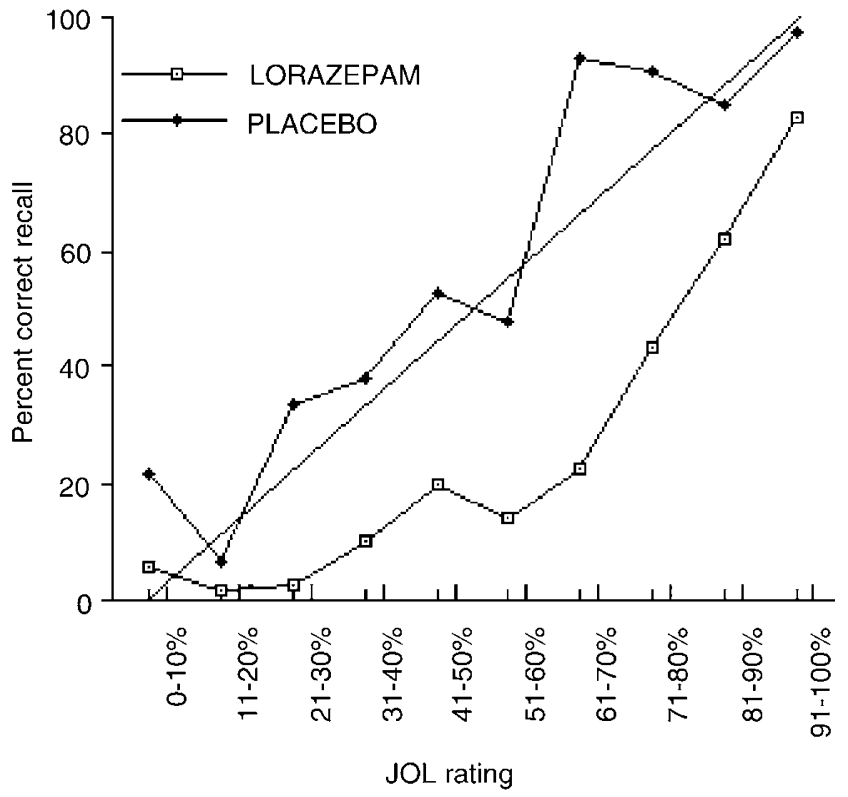

Figure I Calibration curves plotting percent correct recall as a function of JOL for lorazepam and placebo group. The diagonal lines indicate perfect calibration.

Table 3 Mean Magnitude of Item-by-Item JOL Judgments for Pairs of Words on Their Ist, 2nd, and 3rd Repetition

Drug group

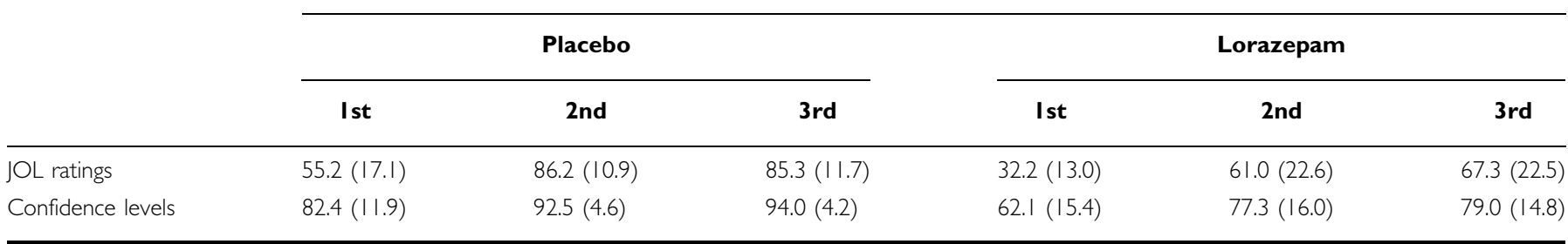

Note: Entries correspond to the means of each individual's mean judgement. 
and association strategies, whereas two participants reported having used no strategy at all. Among the lorazepam participants, only five reported mental imagery with visualization of the words, five participants reported association strategies, one participant reported using mental repetition, and finally, one reported having used no strategy. The last participants reported having adopted inefficient strategies.

\section{DISCUSSION}

The study presented here was specifically designed to examine the effects of presentation frequency on the memory and metamemory performances of lorazepamtreated participants. More precisely, we were interested in investigating the effects of lorazepam on: (1) the way individual participants benefit from repetition of to-belearned items, (2) the accuracy of the monitoring of their learning abilities after encoding, and (3) the control of the study time allocation.

As expected, we observed a deleterious effect of lorazepam on memory performance, lorazepam-treated participants producing fewer correct answers and more numerous commission errors. Nevertheless, in terms of explicit memory performances, there was a clear benefit of repetition for both the lorazepam-treated participants and their controls, the pairs of words presented more frequently being recalled better. There are some other evidences in the literature of conditions under which lorazepam can facilitate the retrieval of material learned when under the effect of the drug (eg File et al, 1999). Also, Mintzer and Griffiths (2001) showed that under the effects of another benzodiazepine, triazolam, memory performance improved when items were simply presented a second time. In our study, both lorazepam and placebo participants benefited from the second repetition. The observed improvement of memory after repetition of the items may be of clinical relevance, as cognitive-function experiments on the longterm effects of benzodiazepines suggest that tolerance to the memory deficits caused by benzodiazepines never fully develops (Gorenstein et al, 1995; Tata et al, 1994). Another observation that may be useful for the cognitive remediation was a difference in the reported use of strategy: most of the placebo participants reported using mental imagery with visualization of the associated words. This was not the case for participants under the effects of lorazepam as they less frequently reported using mental imagery. However, imagery is known to be a superior strategy in such memory tasks. Hence, this could contribute to explain the reported group differences in learning performances. Explicitly suggesting to benzodiazepine consumers the use of such a learning strategy could help these individuals overcome at least some of their memory deficits.

With respect to control and monitoring of memory, results revealed that the two groups differed significantly with regard to the effects of presentation frequency during the encoding phase of the task. Nevertheless, the comparison between study time and JOL is difficult because the former one was immediate and the latter was delayed. Indeed, placebo participants showed an effect of repetition for both the study time and their explicit judgments of how well they had encoded the items (JOL ratings). This was not the case for the lorazepam participants: while the explicit JOL ratings were as sensitive to presentation frequency as that observed in placebo participants, lorazepam-treated individuals did not adapt the time allocated to the study of the various items in function of the presentation frequency. Indeed, they spent as much time studying pairs presented once, twice, or three times. There are several possible explanations to such a behavior: it is possible that the anterograde amnesic effect of lorazepam exerted its action during the course of the experiment itself. In that case, lorazepam participants would have progressively forgotten that they had seen a given pair, and would systematically consider an item as if it were the first time they saw it. This would explain why the time allocated to the learning did not differ with the presentation frequency. This is likely to occur as clinical observations have revealed that benzodiazepine-treated participants usually have no clear awareness of the amnesic effect in progress (Hinrichs et al, 1982; Curran et al, 1987; Weingartner et al, 1993). However, the results reported here indicated that for the JOL delayed ratings, that is, the JOLs reported after the encoding phase, the lorazepam participants did in fact take into account the effect of repetition. Consequently, another explanation may be favored.

Under the effects of lorazepam, participants may be aware that they require more time to correctly acquire information that is to be learned. The absence of control over the study time would then be an optimized strategy, as it would allow for an increase in memory performance regardless of the presentation frequency of the pairs of words. At the same time, the other metacognitive data collected seemed to reveal a relatively good insight of participants under lorazepam about their true cognitive capabilities: when asked for a global evaluation, at the end of the experimental session of the difficulty of the task and of their general performance level, the participants under lorazepam were more numerous than the placebo participants to judge the task as difficult, and most of them considered that their performance was average or even bad, whereas the placebo participants were overall more optimistic. This seems to contradict the clinical observations of a general unawareness of the transitory memory difficulties under the effects of lorazepam (Hinrichs et al, 1982; Curran et al, 1987; Weingartner et al, 1993). However, the fact that participants were asked during the course of the cognitive task to monitor how well they had acquired the material could have improved the participants' general insight of their true cognitive abilities. Also, it must be noted that if lorazepam participants made a different estimation than the placebo participants of the difficulty of the task, this was not a relative estimation, as they were simply asked to rate the difficulty of the task at hand, and not to compare their performance with and without the drug intake. In the present study, it was not possible to infer from the participants' comments if they had in fact been aware of item repetition. Subsequently, for the lorazepam participants, the nondecreased allocation of study time from the 1 st to the 2 nd and 3rd trials could be the consequence of awareness of difficulty and or lack of awareness of item repetition. Finally, if the peculiar way of controlling learning time allocation in the lorazepam participants results indeed 
from a strategical regulation of memory, it is not necessarily accompanied by conscious awareness: there is some evidence in the literature that shows that strategies can be adopted without conscious awareness (Siegler and Stern, 1998). Consequently, the fact that the JOLs expressed by the lorazepam participants were sensitive to item repetition could be an argument in favor of the hypothesis that participants do take into account the fact that they have seen a word-pair more often, but this could have occurred without the individuals being aware of it. As a result of the anterograde amnesic effect of the drug, lorazepam participants would have no memory of the previous presentations, but their metamemory ratings would be coherent with their present state of enhanced difficulty of encoding, as their JOLs are lower than that expressed by the placebo participants, and their monitoring is also coherent with the effect of repetition, as their JOLs increase with item repetition.

There would be a dissociation between memory impairments and metamemory. If a dissociation at encoding between the strategic regulation of learning and monitoring could be observed, the effects of lorazepam resulted in a subtle mixture of preservations and of impairments of cognitive and metacognitive abilities. Our results suggest that the monitoring measure used in the present memory task is not activated in the control aspect of the encoding phase. Of course, whereas study time reflects those processes involved during item study, the delayed JOLs clearly are not. The delayed JOLs could be based on different mnemonic cue from the one of the study time.

Our results further revealed that JOL ratings, even if as accurate at predicting the correctness of the recalled answers, are reduced overall in magnitude under the effects of the lorazepam drug. These results replicate earlier studies, which reported that CLs expressed at the time of retrieval were lower under the effects of the lorazepam drug than in a placebo group, in a task assessing episodic memory (Bacon et al, 1998). However, in this study, the magnitude of the prediction of the FOK with regard to the future retrievability of items that they had failed to recall was not found to be affected by lorazepam intake. Nelson and collaborators obtained similar contrasting results when examining alcohol intoxication (Nelson et al, 1986, 1998): JOL ratings were reduced by alcohol intoxication (Nelson et al, 1998), whereas FOK judgments were not (Nelson et al, 1986). This pattern of results may be due to the fact that JOL ratings and FOK judgments, which are expressed at different steps of a memory operation, are underlined by different mechanisms (Leonesio and Nelson, 1990). Another possibility is that they were measured in different types of behavioral tasks (pairs of word encoding and sentence completion, respectively). In the same vein, Dunlosky et al (1998) investigated how metamemory is affected by inhalation of $\mathrm{N}_{2} \mathrm{O}$. This gas has been shown to produce a variety of effects, including analgesia and anxiolysis as well as memory impairments. Inhaling $\mathrm{N}_{2} \mathrm{O}$ during the encoding phase of a learning task was shown to reduce subsequent recall performances compared to that observed when participants inhaled a placebo gas. In contrast, the accuracy of people's JOLs was at least as precise for people who had inhaled $\mathrm{N}_{2} \mathrm{O}$ than for those who had inhaled placebo. Hence, one may conclude that $\mathrm{N}_{2} \mathrm{O}$ significantly affects subsequent recall performances without affecting the accuracy of JOL ratings - even if the overall level of delayed JOL ratings was shown to be reduced. For lorazepam compared to placebotreated participants similar results were obtained: we report impaired recall, similar accuracy of the JOL ratings together with overall lower JOL ratings. The similarity of the results obtained in the present study and in the study where subjects inhaled $\mathrm{N}_{2} \mathrm{O}$ confirms the implication of the GABA/ benzodiazepine nervous transmission in the effects of these drugs on cognition and metacognition.

In opposition to these results come those by Moulin et al (2000b). These authors report a series of experiments that suggest a dissociation between the mnemonic bases of metamemory control and metamemory monitoring during the encoding phase of a memory task (Moulin et al, 2000a, b, 2001, 2002a, b). Their work suggests that for Alzheimer patients a dissociation is revealed between monitoring and control processes. In their studies, Alzheimer patients allocated less time to learn repeated items (eg the control aspect) but their immediate JOL ratings (immediately after the presentation of one pair) were insensitive to repetition frequency. Hence, in these patients, control mechanisms were 'relatively' normal (with respect to the behavior of healthy participants), whereas the monitoring processes were abnormal. The elderly (healthy) control participants made anticipated immediate JOL ratings that were affected by the presentation frequency, as did young healthy individuals: immediate JOL ratings were higher for items seen on the third occasion than when they were first seen. An important point when compared to the present study is the fact that both Alzheimer and elderly control participants significantly reduced the study time for items that had been seen more often. Thus, while the Alzheimer group showed a performance pattern for study time similar to that of healthy participants, whether young or elderly, their explicit immediate JOL ratings of how well they had encoded the items was not. Such a profile of impairment, which differs from that observed during the pharmacologically induced amnesic episodes, seems to suggest a lower implication of the GABA/benzodiazepine system in the cognitive and metacognitive impairments occurring in Alzheimer's disease. At least point, we must specify that for the Moulin's studies only immediate JOL ratings were evaluated; in our study, we measured delayed JOLs. It is possible that with item repetition, the anterograde amnesic effects of lorazepam would have allowed participants to improve their performances in terms of both the memory of the target item and the accumulation of mnemonic cues, which would be revealed through increased JOL ratings.

Finally, the observation of another profile of memory and metamemory impairments during encoding in patients with schizophrenia (Bacon and Izaute, 2004) leads to suggest that different patterns of cognitive and metacognitive impairments may occur relative to JOL ratings, as it has previously been observed with another metamemory judgement, namely the FOK (Shimamura and Squire, 1984, 1986; Pappas et al, 1992). The results also suggest that various nervous transmission systems are implicated differentially in the diverse patterns of memory and metamemory impairments that may occur in humans. 


\section{ACKNOWLEDGEMENTS}

This work was supported by a Cognitique Grant (No. COG 53B) 'Perturbations and recovery of cognitive functions' from the French Ministry of Research. We thank Christine Ramana-Keller for technical assistance and Dr Marie Welsch for the medical examination of the participants. We particularly thank Yvonne Delevoye-Turrell who patiently corrected our English. We also thank the INSERM and the CNRS institutions. We also thank Martin Keller and three anonymous reviewers for their very helpful comments on an earlier version of this article.

\section{REFERENCES}

Allen D, Curran HV, Lader M (1993). The effects of single doses of CL 284, 846, lorazepam, and placebo on psychomotor and memory function in normal male volunteers. Eur J Clin Pharmacol 45: 313-320.

Bacon E, Danion JM, Kauffman-Muller F, Schelstraete MA, Bruant A, Sellal F et al (1998). Confidence level and feeling of knowing for episodic and semantic memory: an investigation of lorazepam effects on metamemory. Psychopharmacology 138: 318-325.

Bacon E, Izaute M (2004). The effects of repetition and difficulty on memory and consciousness in patients with schizophrenia. Schizophr Res 67: 270.

Bishop K, Curran V (1998). An investigation of the effects of benzodiazepine receptor ligands and of scopolamine on conceptual priming. Psychopharmacology 140: 345-353.

Blin O, Simon N, Jouve E, Habib M, Gayraud D, Durand A et al (2001). Pharmacokinetic and pharmacodynamic analysis of sedative and amnesic effects of lorazepam in healthy volunteers. Clin Neuropharmacol 24: 71-81.

Bond A, Lader M (1974). The use of analog scales in rating subjective feelings. Br J Med Psychol 47: 211-218.

Curran HV (1991). Benzodiazepines, memory and mood: a review. Psychopharmacology 105: 1-8.

Curran HV (1999). Effects of anxiolytics on memory. Hum Psychopharmacol 14: 72-79.

Curran HV, Gardiner JM, Java RI, Allen D (1993). Effects of lorazepam upon recollective experience in recognition memory. Psychopharmacology 110: 374-378.

Curran HV, Schiwy W, Lader M (1987). Differential amnesic properties of benzodiazepines: a dose-response comparison of two drugs with similar elimination half-lifes. Psychopharmaco$\log y$ 92: 358-364.

Danion JM (1994). Drugs as tools for investigating memory. Eur Neuropsychopharmacol 4: 179-180.

Danion JM, Peretti S, Grange D, Bilik M, Imbs JL, Singer L (1992). Effects of chlorpromazine and lorazepam on explicit memory, repetition priming and cognitive skill learning in healthy volunteers. Psychopharmacology 108: 345-351.

Danion JM, Zimmermann MA, Willard-Schroeder D, Grangé D, Singer L (1989). Diazepam induces a dissociation between explicit and implicit memory. Psychopharmacology 99: 238-243.

Duka T, Curran HV, Rusted JM, Weingartner HJ (1996). Perspectives on cognitive psychopharmacology research. Behav Pharmacol 7: 401-410.

Dunlosky J, Domoto PK, Wang ML, Ishikawa T, Roberson I, Nelson TO et al (1998). Inhalation of $30 \%$ nitrous oxide impairs people's learning without impairing people's judgments of what will be remembered. Exp Clin Psychopharmacol 6: 77-86.

Dunlosky J, Nelson TO (1994). Does the sensitivity of judgments of learning (JOLs) to the effects of various study activities depend on when the JOLs occur? J Mem Lang 33: 545-565.
File S, Fluck E, Joyce E (1999). Conditions under which lorazepam can facilitate retrieval. J Clin Psychopharmacol 19: 349-353.

File SE, Sharma R, Shaffer J (1992). Is lorazepam-induced amnesia specific to the type of memory or to the task used to assess it? J Psychopharmacol 6: 76-80.

Fluck E, File SE, Springett J, Kopelman MD, Rees J, Orgill J (1998). Does the sedation resulting from sleep deprivation and lorazepam cause similar cognitive deficits? Pharmarcol Biochem Behav 59: 909-915.

Gonzales RA, Hoffman PL (1991). Receptor-gated ion channels may be selective CNS targets for ethanol. Trends Pharmacol Sci 1: $1-3$.

Gorenstein C, Bernik MA, Pompéia S, Marcourakis T (1995). Impairment of performance associated with long-term use of benzodiazepines. J Psychopharmacol 9: 313-318.

Green JF, McElholm A, King DJ (1996). A comparison of the sedative and amnestic effects of chlorpromazine and lorazepam. Psychopharmacology 128: 67-73.

Hinrichs JV, Mewaldt SP, Ghoneim MM, Berie JL (1982). Diazepam and learning: assessment of acquisition deficits. Pharmacol Biochem Behav 17: 165-170.

Huron C, Giersch A, Danion JM (2002). Lorazepam, sedation and conscious recollection: a dose-response study with healthy volunteers. Int Clin Psychopharmacol 17: 19-26.

Kelemen WL, Weawer III CA (1997). Enhanced metamemory at delays: why do judgements of learning improve over time? J Exp Psychol: Learn Mem Cognition 23: 1394-1409.

Kimball DR, Metcalfe J (2003). Delaying judgments of learning affects memory, not metamemory. Mem Cognition 31: 918-929.

Koriat A (1997). Monitoring one's knowledge during study: a cue utilization approach to judgments of learning. J Exp Psychol: General 126: 349-370.

Koriat A, Goldsmith M (1996). Monitoring and control processes in the strategic regulation of memory accuracy. Psychol Rev 103: 490-517.

Legrand F, Vidailhet P, Danion JM, Grangé D, Giersch A, Van der Linden $\mathrm{M}$ et al (1995). Differential effects of diazepam and lorazepam on repetition priming on healthy volunteers. Psychopharmacology 118: 475-479.

Leonesio RJ, Nelson TO (1990). Do different metamemory judgments tap the same underlying aspects of memory? J Exp Psychol: Learn Mem Cognition 16: 464-470.

Lichenstein S, Fischhoff B, Phillips LD (1982). Calibration of probabilities: the state of the art to 1980. In: Kahneman D, Slovic P, Tversky A (eds). Judgment Under Uncertainty: Heuristics and Biases. Cambridge University Press: Cambridge, England. pp 306-334.

Mazzoni G, Nelson TO (1995). Judgments of learning are affected by the kind of encoding in ways that cannot be attributed to the level of recall. J Exp Psychol: Learn Mem Cognition 21: 1263-1274.

Merritt P, Hirshman E, Hsu J, Berrigan M (2003). Metamemory without the memory: the effect of midazolam on judgments of learning. Abstr Psychonomic Soc 8: 92.

Mintzer MZ, Griffiths RR (2001). False recognitions in triazolamindiced amnesia. J Mem Lang 44: 475-492.

Mintzer MZ, Griffiths RR (2003a). Lorazepam and scopolamine: a single-dose comparison of effects on human memory and attentional processes. Exp Clin Psychopharmacol 11: 56-72.

Mintzer MZ, Griffiths RR (2003b). Dose-effect comparison of lorazepam and scopolamine on metamemory. Effects on human memory and attentional processes. Abstr Psychonomic Soc 8: 92.

Moulin CJA, Perfect TJ, Conway MA, North AS, Jones RW, James N. (2002a). Retrieval-induced forgetting in Alzheimer's disease. Neuropsychologia 40: 862-867.

Moulin CJA, Perfect TJ, Fitch F (2002b). Metacognitive processes at encoding: insights from Alzheimer's disease. In: Chambres P, Izaute M, Marescaux PJ (eds). Metacognition: Process, Function, 
and Use. Kluwer Academic Publishers: Dordretch, the Netherlands. pp 91-101.

Moulin CJA, Perfect TJ, Jones RW (2000a). Evidence for intact memory monitoring in Alzheimer's disease: metamemory sensitivity at encoding. Neuropsychologia 38: 1242-1250.

Moulin CJA, Perfect TJ, Jones RW (2000b). The effects of repetition on allocation of study time and judgments of learning in Alzheimer's disease. Neuropsychologia 38: 748-756.

Moulin CJA, Perfect TJ, Jones RW (2001). Global predictions of memory in Alzheimer's disease: Evidence for preserved metamemory monitoring. Aging Neuropsychol Cognition 7: 230-244.

Nelson TO (1984). A comparison of current measures of the accuracy of feeling-of-knowing predictions. Psychol Bull 95: $109-133$.

Nelson TO (1987). The Goodman-Kruskal gamma coefficient as an alternative to signal-detection theory's measures of absolutejudgement accuracy. In: Roskam E, Suck R (eds). Progress in Mathematical Psychology. Amsterdam: Elsevier/North-Holland. pp 299-306.

Nelson TO (1993). COMMENTS judgments of learning and the allocation of study time. J Exp Psychol: General 122: 269-273.

Nelson TO (1996a). Consciousness and metacognition. Am Psychol 51: $102-116$

Nelson TO (1996b). Gamma is a measure of the accuracy of predicting performance on one item relative to another item, not of the absolute performance on an Individual Item. Comments on Shraw (1995). Appl Cognitive Psychol 10: 257-260.

Nelson TO, Dunlosky J (1991). When people's judgments of learning (JOLs) are extremely accurate at predicting subsequent recall: The 'delayed-JOL effect'. Psychol Sci 2: 267-270.

Nelson TO, Dunlosky J, Graf A, Narens L (1994). Utilization of metacognitive judgments in the allocation of study during multitrial learning. Psychol Sci 5: 207-213.

Nelson TO, Graf A, Dunlosky J, Marlatt A, Walker D, Luce K (1998). Effect of acute alcohol intoxication on recall and on judgments of learning during the acquisition of new information. In: Mazzoni G, Nelson TO (eds). Metacognition and Cognitive Neuropsychology, Monitoring and Control Processes. LEA: Mahwah, NJ. pp 161-180.

Nelson TO, McSpadden M, Fromme K, Marlatt GA (1986). Effects of alcohol intoxication on metamemory and on retrieval from long-term memory. J Exp Psychol: General 115: 247-254.
Nelson TO, Narens L (1990). Metamemory: a theoretical framework and new findings. In: Bower GH (ed). The Psychology of Learning and Motivation. Academic Press: New York. pp 1-45.

Norris H (1971). The action of sedatives on brain stem occulomotor system in man. Neuropharmacology 10: 181-191.

Pappas BA, Sunderland T, Weingartner HM, Vitiello B, Martinson $\mathrm{H}$, Putnam K (1992). Alzheimer's disease and feeling-of-knowing for knowledge and episodic memory. J Gerontol: Psychol Sci 47: 159-164.

Patat A, Perault MC, Vandel B, Ulliac N, Zieleniuk I, Rosenzweig J. (1995). Lack of interaction between a new antihistamine, mizolastine, and lorazepam on psychomotor performance and memory in healthy volunteers. Br J Clin Pharmacol 39: 31-38.

Pompéia S, Gorenstein C, Curran HV (1996). Benzodiazepine effects on memory tests: dependence on retrieval cues? Int Clin Psychopharmacol 11: 229-236.

Shimamura AP, Squire LR (1986). Memory and metamemory: a study of the feeling-of-knowing phenomenon in amnesic patients. J Exp Psychol: Learn Mem Cognition 12: 452-460.

Shimamura AP, Squire LR (1984). Paired-associate learning and priming effects in amnesia: a Neuropsychological Study. J Exp Psychol: General 113: 556-570.

Siegler RS, Stern E (1998). Conscious and unconscious strategy discoveries: a microgenetic analysis. J Exp Psychology: General 127: 377-397.

Tata PR, Philip R, Rollings J, Collins M, Pickering A, Jacobson RR (1994). Lack of cognitive recovery following withdrawal from long-term benzodiazepine use. Psychol Med 24: 203-213.

Ticku MK (1983). Benzodiazepine-GABA receptor-ionophore complex. Curr Concepts. Neuropharmacology 22: 1459-1470.

Vermeeren A, Jackson JL, Muntjewerff ND, Quint PJ, Harrison EM, O'Hanlon JF (1995). Comparison of acute alprazolam $(0.25,0.50$ and $1.0 \mathrm{mg}$ ) effects versus those of lorazepam $2 \mathrm{mg}$ and placebo on memory in healthy volunteers using laboratory and telephone tests. Psychopharmacology 118: 1-9.

Vidailhet P, Kazès M, Danion JM, Kauffmann-Muller F, Grangé D (1996). Effects of lorazepam and diazepam on conscious and automatic memory processes. Psychopharmacology 127: 63-72.

Wechsler D (1987). Wechsler Memory Scale, Revisited Manual. Psychological Corporation: San Antonio, TX.

Weingartner HJ, Joyce EM, Sirocco KY, Adams CM, Eckardt MJ, George T et al (1993). Specific memory and sedative effects of the benzodiazepine triazolam. J Psychopharmacol 7: 305-315. 